\title{
Uma introdução à visualização de informações
}

HUGO A. D. DO NASCIMENTO

CRISTIANE B. R. FERREIRA

\section{Resumo}

Visualização de Informações é uma área emergente da Ciência que estuda formas de apresentar dados abstratos visualmente de tal modo que relações entre os mesmos são melhor compreendidas ou novas informações possam ser descobertas. O presente artigo discute vários aspectos relacionados à apresentação visual de dados e introduz técnicas efetivas e bem conhecidas para visualização de informações. As técnicas estudadas aqui têm aplicações práticas nos diversos campos da Ciência. O trabalho também aborda alguns dos desafios atuais da área de Visualização de Informações.

Palavras-chave:

Dados abstratos, apresentação de informações, descoberta de informações 


\title{
An introduction to information visualization
}

\author{
HUGO A. D. DO NASCIMENTO \\ CRISTIANE B. R. FERREIRA
}

\begin{abstract}
Information Visualization is an emerging scientific field that studies ways of presenting abstract data visually, so that information about that data can be better understood or even discovered. This paper discusses several aspects regarding the visual presentation of data and introduces well known and effective techniques for information visualization. The techniques presented here have applications in many scientific fields. The paper also discusses some of the modern challenges in the Information
\end{abstract} sentation, information discovery 


\section{Introdução}

A área de Visualização de Informações é um campo emergente de trabalho que se preocupa com a construção de representações visuais de dados abstratos. O processo de visualização envolve a transformação desses dados em imagens mentais ou reais que possam ser visualizadas pelos seres humanos. $\mathrm{O}$ objetivo é auxiliar no entendimento de determinado assunto, o qual, sem uma visualização, exigiria maior esforço para ser compreendido. Em alguns casos, a meta da visualização é ajudar também na descoberta de novas informações, "escondidas" nos dados abstratos.

Exemplos de visualizações de informações são muito comuns principalmente na forma de gráficos de linhas, de barras e de pizza. Esses tipos de visualizações simples são utilizados em livros didáticos e aparecem com frequência em revistas, jornais impressos e em noticiários de TV, por exemplo, para apresentar pesquisas de opinião.

Outras técnicas de visualizações de informações mais complexas foram desenvolvidas e têm sido empregadas em uma gama de aplicações práticas, tais como: no monitoramento de bolsas de valores (DWYER; EADES, 2002), na consulta a bases de dados (SHNEIDERMAN, 1994), e no desenho de diagramas como organogramas e árvores genealógicas (DI BATTISTA et al., 1999).

O presente artigo introduz o leitor na vasta e interessante área de Visualização de Informações. A Seção 2 discute as vantagens de utilizar visualizações para apresentar dados abstratos. A Seção 3 descreve um processo para gerar visualizações, discorre sobre aspectos relacionados a efetividade e eficiência das visualizações e a vinculação desse tema com outras áreas do conhecimento. A Seção 4, por sua vez, categoriza as técnicas de visualização e apresenta algumas técnicas bem conhe- 
Figura 1: reconhecimento de padrões. Os leitores conseguem localizar rapidamente a estrela no meio dos quadrados, conhecendo previamente seus possíveis formatos. Se a estrela estivesse pintada com uma cor mais escura do que a dos demais símbolos, então o processo de reconhecimento seria ainda mais fácil. cidas embora não triviais. Por fim, a Seção 5 conclui com uma análise geral do assunto e com tema para trabalhos futuros nesta área.

\section{O Porquê de Visualizar}

A utilização de técnicas de visualização de informações para ampliar a cognição sobre dados abstratos tem um forte apelo quando comparada com outras formas de transmitir e/ou de analisar informações.

Em primeiro lugar, uma grande quantidade de dados pode ser condensada em uma simples visualização. Isso porque o processo de visualização envolve o sentido humano que possui maior capacidade de captação de informações por unidade de tempo: a visão. Esse sentido é rápido e paralelo e permite, inclusive, prestar atenção em um objeto de interesse sem perder de vista o que está acontecendo ao seu redor (obviamente, com menos detalhes). O sistema visual humano também é treinado para reconhecer padrões, podendo identificar formas e cores diferentes e, assim, localizar objetivos específicos em uma imagem (essa capacidade é ilustrada na Figura 1).

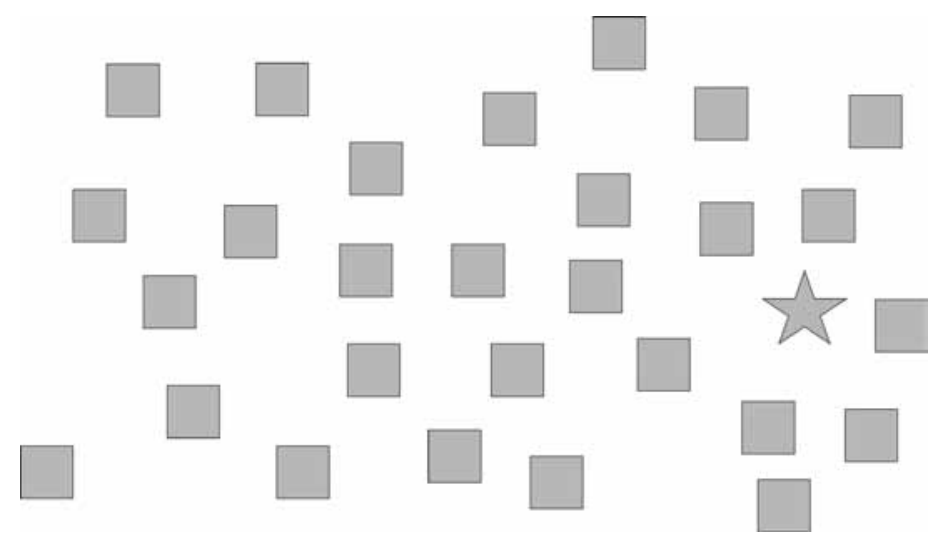

Tais características do sistema visual permitem identificar e lidar com situações complexas do dia a dia que envolvem processamento visual.

Não menos importante, as visualizações, por si só, trazem benefícios, uma vez que podem funcionar como uma exten- 
são da memória humana e como auxílio ao processo cognitivo. É comum a escrita de anotações em uma agenda ou em um calendário para lembrar as pessoas de assuntos a serem discutidos ou de eventos que ocorreram ou que irão ocorrer. Também é frequente, para estudantes e profissionais, o desenho de diagramas e a organização de informações espacialmente em uma folha de papel quando estudando um problema que envolve diversas partes. Essas imagens ajudam a entender o problema e/ou a encontrar uma solução para o mesmo, além de facilitar a memorização do objeto em estudo.

\section{Definições Básicas, Modelo para Visualiza- ção e Áreas Afins}

Card e outros (CARD et al., 1999) definem Visualização de Informações como sendo "o uso de representações visuais de dados abstratos suportadas por computador e interativas para ampliar a cognição". É importante notar o papel moderno da definição desses autores ao incluir a ferramenta computacional com suporte natural ao processo de apresentar e interagir com visualizações, em contraposição a visualizações antigas (da época pré-computacional) as quais quase sempre eram estáticas, sem interatividade.

Deve-se observar também que o estudo de Visualização de Informações difere de uma área similar chamada Visualização Científica. Ambas as áreas têm como objetivo produzir visualizações que ampliem a percepção e o entendimento das informações embutidas nas imagens, levando à aquisição e solidificação do conhecimento. No entanto, na Visualização Científica, os dados a serem apresentados geralmente correspondem a medidas de objetos físicos, fenômenos da natureza ou posições em um domínio espacial, possuindo, assim, uma representação geométrica intrínseca. Como exemplo de visualizações nessa linha, podemos citar a visualização de órgãos do corpo humano, de fluidos em movimento e, até mesmo, de funções matemáticas. Já em uma Visualização de Informações, os dados são abstratos, não havendo necessariamente uma representação geométrica inerente aos mesmos. Neste caso, uma imagem deve ser gerada com base nos relacionamentos ou informações que podem ser inferidos acerca dos dados e na experiência do usuário de apresentar dados desse tipo.

A Figura 2 ilustra um modelo de referência para o processo de visualização de informações, apresentado por Card 
Figura 2: Modelo de Referência para Visualização de Informações e outros (CARD et al., 1999), o qual é formado por três etapas conforme descritas a seguir.

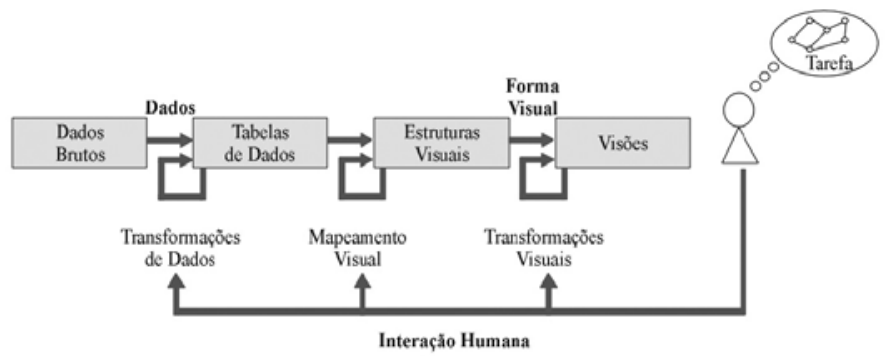

A primeira etapa é chamada de Transformações de Dados. Na mesma, um conjunto de dados brutos é processado e organizado em uma representação lógica mais estruturada, geralmente na forma de uma ou mais tabelas. O processamento pode envolver a eliminação de dados redundantes, errados ou incompletos, bem como a filtragem e o agrupamento dos dados relevantes. Além disso, pode ser feita a inclusão de novas informações, como, por exemplo, de resultados de análises estatísticas (média, soma total, desvio padrão e etc.) realizadas sobre os dados brutos. Uma forma comum de organizar os dados em tabelas é alocar uma linha para cada dado e uma coluna para cada atributo diferente do mesmo. Neste caso, a quantidade de linhas indicará o número total de dados a serem visualizados e o total de colunas representará a dimensão dos dados.

A próxima etapa é o Mapeamento Visual, a qual envolve a construção de uma estrutura visual que represente visualmente os dados da tabela. Toda estrutura visual pode ser decomposta em três partes: substrato espacial, marcas visuais e propriedades gráficas das marcas.

O substrato visual caracteriza o espaço para a visualização, sendo normalmente representado por eixos tais como os eixos X e Y do plano cartesiano. Há quatro tipos elementares de eixos:

- $U$ = eixo não estruturado (ou sem eixo);

- $\mathrm{N}$ = eixo nominal (região dividida em sub-regiões);

- $\mathrm{O}=$ eixo ordenado (região dividida em sub-regiões onde a ordem das mesmas tem importância);

- $\mathrm{Q}$ = eixo quantitativo (região que tem uma métrica, como valores inteiros ou reais). 
As marcas visuais são símbolos gráficos utilizados para representar os itens de dados. A Figura 3 demonstra os tipos de marcas visuais mais comuns, sendo eles pontos (figuras geométricas simples com a finalidade de marcar uma posição), linhas, áreas, volumes e figuras complexas (cujos detalhes têm importância; também chamadas de ícones ou glyphs).

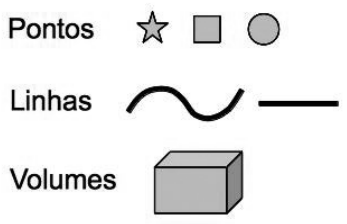

Áreas

Figuras

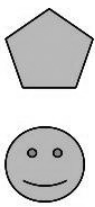

Já as propriedades gráficas são os atributos visíveis que caracterizam as marcas visuais. Algumas propriedades amplamente utilizadas são: a posição da marca dentro do substrato espacial; a forma, a cor, o tamanho, e a área ou o volume da marca; a orientação, o sentido e a inclinação de marcas do tipo de linha; e a textura de uma marca na forma de área. Certas propriedades gráficas dão um aspecto de dinamismo às marcas e servem para destacar atributos dos dados que necessitam de maior atenção do usuário. São exemplos dessas propriedades a possibilidade de animação ou de movimento da marca, a velocidade, a direção e o sentido do seu deslocamento, e a frequência com que uma marca altera sua forma ou posição na tela.

No Mapeamento Visual, um substrato espacial deve ser escolhido e marcas visuais associadas aos itens de dados abstratos dentro do substrato. Cada atributo dos dados deve ser vinculado, por sua vez, a uma ou mais propriedades gráficas das marcas. Um exemplo de mapeamento visual seria o da construção de um gráfico simples de linhas representando a evolução mensal de vários itens de despesas familiares. As dados de interesse poderiam estar cadastrados em um tabela com uma despesa por linha, as colunas representado os meses do ano e cada célula contendo o valor da respectiva despesa em um dado mês. O substrato espacial, neste caso, poderia conter um eixo quantitativo Y, representando valores em Real, e um eixo ordinal $\mathrm{X}$, com os meses. Os itens de dados, descritos por linhas da tabela, seriam associados a linhas gráficas na figura. Já os atributos desses dados, isto é o nome da despesa
Figura 3: Tipos de marcas visuais. 
e o seu valor em cada mês, poderiam ser associados à cor e à altura da linha em seu diversos pontos, respectivamente.

A última etapa do processo de visualização é a de Transformações Visuais, na qual é possível modificar e estender as estruturas visuais interativamente através de operações básicas como:

- testes de localização, que possibilitam obter informações adicionais sobre um item da tabela de dados;

- controles de ponto de vista, os quais permitem ampliar, reduzir e deslocar a imagem com o objetivo de oferecer visões diferentes; e

- distorções da imagem, visando criar ampliações de uma região específica em detrimento de outra.

Os mecanismos de interação implementados nessa etapa possibilitam ao usuário explorar diferentes cenários para um melhor entendimento dos dados visualizados. Além disso, o esforço de exploração dos dados é repassado em parte para o computador, uma vez que os cálculos e o redesenho da imagem são realizados pela máquina, deixando para o usuário a tarefa de observar o que acontece quando a visualização se modifica.

O processo descrito acima poderia ser chamado de "Visualização de Dados", uma vez que a imagem gerada está diretamente relacionada com os dados de origem. Mesmo assim, prefere-se a utilização do termo "Visualização de Informações" uma vez que o foco do processo é em compreender as informações acerca da relação entre os dados, mais do que observar seus valores isolados propriamente ditos.

É importante ressaltar que nem todo exemplo de visualização de informações é útil para o usuário. Em geral, dois aspectos de uma visualização nos ajudam a determinar a sua provável utilidade. Esses aspectos, apresentados por Mackinlay (1986) como critérios para avaliar linguagens gráficas, são a expressividade e a efetividade.

Uma visualização pode ser considerada expressiva se ela é capaz de mostrar todos os dados de interesse do usuário e nada mais.

Já a efetividade está relacionada com a apresentação das informação de forma clara. Para ser efetiva, uma visualização deve ser de fácil e rápida percepção e induzir a uma quantidade menor de erros de interpretação do que outras formas de se visualizar os mesmos dados.

Expressividade e efetividade são aspectos importantes porque, sem os mesmos, uma visualização pode não ser ca- 
paz de enfatizar padrões relevantes nos dados, não trazendo, assim, quaisquer informações novas além daquilo que já é trivialmente conhecido. Além disso, uma visualização também pode ser de difícil entendimento ou, até mesmo, sugerir interpretações errôneas, que na verdade não condizem com o significado dos dados.

Os problemas mais comuns que comprometem a efetividade de uma visualização são:

- não colocar dados suficientes na visualização de forma a contextualizar as informações mais relevantes apresentadas;

- desconsiderar atributos importantes dos dados;

- utilizar gráficos sobrepostos em escalas diferentes ou com sistemas de coordenadas distintos, o que impede uma comparação justa entre os dados; e

- não fazer um mapeamento adequado dos dados para marcas e atributos visuais.

Obviamente, características como cor, dimensionalidade, luminosidade, tamanho e forma dos objetos são fatores que auxiliam no processo de cognição e que podem ser explorados na construção de visualizações efetivas. Outros aspectos como a existência de mecanismos de interação com os dados e a possibilidade de compactar uma grande quantidade de informações úteis em uma mesma imagem também podem contribuir para melhorar a visualização.

Algumas orientações para aumentar a expressividade e efetividade das visualizações incluem:

- utilizar o modelo de visualização apresentado na Figura 2 no desenvolvimento de novas visualizações. Esse modelo ajuda na modularização do processo de transformação de dados brutos em imagens interativas. Com isso, novos recursos podem ser adicionados à visualização sem a necessidade de alterar todo o processo;

- eliminar da visualização gráficos e textos desnecessários. Uma boa medida de quantos elementos são desnecessários em uma figura é a quantidade de tinta ou de pixels gastos com itens que não são dados de interesse;

- buscar simplicidade. Isto é, entre duas formas diferentes de representar visualmente uma mesma informação, preferir a mais simples;

- aumentar a quantidade de dados por centímetro quadrado. Apesar de parece contraditório com a orientação anterior, a inclusão de mais dados de interesse em uma imagem, sem sobrecarregá-la demasiadamente, pode ajudar na compreensão do problema como um todo; 
- explorar a utilização de símbolos e de atributos visuais que facilitem a percepção dos dados e dos padrões existentes nos mesmos. Por exemplo, é possível destacar elementos importantes em uma imagem através do uso de uma cor mais chamativa ou colocando informações piscando na tela. Certas cores e símbolos gráficos também já são bem conhecidos culturalmente por terem um significado específico; essas cores e símbolos podem ser associados a dados abstratos para transmitir-lhes um significado similar;

- permitir formas de interação com a visualização; e

- utilizar animações gradativas, sempre que necessário mudar uma visualização na tela, de forma a preservar o "mapa mental" (onde e como estão localizados visualmente os dados) que o usuário tem da imagem. Se uma visualização muda drasticamente, pode ser difícil para o usuário entender o que aconteceu, já que o seu mapa mental não coincide com a nova representação visual.

No que diz respeito à associação de atributos de dados a tais atributos visuais, Mackinlay (1986) propõe ordens de prioridade para se utilizar tais atributos visuais. As ordens de prioridade de Mackinlay, as quais são uma extensão do trabalho desenvolvido por Clevelan e McGill (1984), são descritas na tabela a seguir para três tipos de dados (quantitativos, ordinais e nominais), indo do atributo visual mais perceptível até o menos perceptível. A ideia é associar os atributos mais importantes dos dados aos atributos visuais no topo da lista, seguindo a sequência recomendada.

\begin{tabular}{|c|l|l|l|}
\hline Percepção & Dados Quantitativos & Dados Ordinais & Dados Nominais \\
\hline \multirow{2}{*}{ Maior } & Posição & Posição & Posição \\
$\mid$ & Comprimento & Densidade & Croma de cor \\
$\mid$ & Ângulo & Saturação de cor & Textura \\
$\mid$ & Inclinação & Croma de cor & Conexão \\
$\mid$ & Área & Textura & Envolvimento \\
$\mid$ & Volume & Conexão & Densidade \\
$\mid$ & Saturação de cor & Envolvimento & Saturação de cor \\
$\mid$ & Croma de cor & Aomprimento & Forma \\
$\mid$ & Textura & Anclinação & Comprimento \\
$\mid$ & Conexão & Ángulo \\
v & Envolvimento & Aolume & Inclinação \\
Menor & Forma & Aorma & Aolume \\
\hline
\end{tabular}


Como exemplo, suponha que se deseje representar visualmente dados quantitativos de pacientes de um hospital, como peso, altura e tempo de internação. De acordo com a Tabela 1, o atributo mais importante deveria ser associados à posição dos elementos visuais na tela. Os demais atributos poderiam ser associados ao comprimento, ângulo e inclinação da marca visual, nesta ordem.

Como Visualização de Informações envolve o ato de ver que, de acordo com Marr (1982), é uma tarefa complexa que abrange a aquisição de imagens do ambiente - e também as tarefas de classificação, armazenamento, consulta, filtragem e apresentação de informações relevantes dentro de um contexto, os estudos sobre o assunto não podem ser aprofundados sem os conhecimento advindos de diversos outros ramos da Ciência. Sendo assim, Visualização de Informações se encontra intimamente relacionada à Psicologia, à Linguística e às Artes Visuais, no que se refere à forma como o ser humano vê e interpreta o que está sendo visto. Ela possui também grande relação com subáreas da Computação, como Computação Gráfica, Visão Computacional, Interação Homem-Computador e Mineração de Dados.

Apesar de ser uma área relativamente nova, existem conferências e simpósios importantes realizados mundialmente sobre Visualização de Informações. Dentre eles, destacamse o ACM Symposium on Software Visualization, o Asian Pacific Symposium on Information Visualisation, o Eurographics / IEEE VGTC Symposium on Visualization, o IEEE Symposium on Information Visualization e o International Conference of Information Visualization. No Brasil, eventos tradicionais como o Brazilian Symposium on Computer Graphics and Image Processing - SIBGRAPI e o Simpósio Brasileiro de Banco de Dados possuem linhas de publicação sobre Visualização de Informações.

Livros especializados também apresentam conceitos e técnicas sobre essa área como os de Tufte (1983, 1990 e 1997), Card e outros (CARD et al., 1999), Spence (2000) e Ware (2004).

\section{Técnicas de Visualização de Informações}

Existem dois critérios principais para o estudo e a classificação das técnicas de visualização de informações. O primeiro considera o modo como as técnicas exploram o substrato visual, as marcas e as propriedades visuais do desenho. Nesse crité- 
rio, temos técnicas que utilizam cores, manipulam a dimensão do substrato visual, modificam dinamicamente a associação dos dados a marcas visuais, variam a escala de ampliação da imagem e empregam figuras metafóricas para apresentar informações. No segundo critério, as técnicas são classificadas de acordo com as características dos dados a serem visualizados. Dessa forma, existem técnicas adequadas para dados unidimensionais, bidimensionais e tridimensionais com atributos aparentemente independentes, dados multi-dimensionais (quatro ou mais dimensões) e para dados conectados em redes (modelados como grafos).

Considerando o primeiro critério, uma abordagem bastante explorada por várias técnicas na apresentação de informações é o emprego de cores. Colocar "a cor certa no lugar certo" não é uma tarefa trivial, uma vez que a associação inapropriada de atributos de dados a cores pode causar uma catástrofe visual. Tufte (1990) e Ware (2004) apresentam alguns princípios e orientações para o uso de cores. Tufte, por exemplo, alerta que, no caso de mapas, cores puras muito fortes ou brilhantes podem resultar em efeitos desagradáveis quando empregadas em grandes áreas adjacentes, mas podem ter efeitos extraordinários se utilizadas de forma reduzida ou entre dois tons de fundo (background). Ware, por sua vez, destaca a preocupação com o contraste da cor de uma marca visual com a cor de fundo, já que o contraste entre cores adjacentes pode alterar significativamente a percepção de uma cor, fazendo com que ela pareça mais clara ou escura. Ware também sugere meios para tentar reduzir esse efeito, como, por exemplo, desenhar uma fina borda branca ou preta ao redor do objeto colorido.

Com relação à dimensão do substrato visual, Card e outros (CARD et al., 1999) fazem uma discussão detalhada. Estruturas unidimensionais são tipicamente empregadas para a apresentação de documentos de texto ou de linhas do tempo, podendo ser combinadas com um segundo ou terceiro eixo para mostrar comparação entre valores. Já estruturas visuais bidimensionais, como gráficos de linhas, barras, pizza e mapas, são mais utilizadas para apresentar dados estatísticos, descrever funções matemáticas e visualizar informações geográficas. Estruturas visuais $3 \mathrm{D}$, por sua vez, são úteis para visualizar dados representando elementos matemáticos ou físicos ou para combinar duas ou mais visualizações $2 \mathrm{D}$.

A Figura 4 mostra uma tela do programa HomeFinder (WILLIAMSON; SHNEIDERMAN, 1992), a qual é também um bom exemplo de visualização 2D. O HomeFinder permite 
filtrar e visualizar uma base de dados sobre casas à venda utilizando uma representação de mapa. No sistema, o usuário realiza consultas dinâmicas à base de dados através de um painel no lado direito da tela, podendo filtrar casas de acordo com a distância a um ponto de referência, preço, ano de construção, área total e quantidade de quartos e de banheiros. As casas que se encaixam no padrão definido pelos filtros são destacadas como pontos coloridos no mapa, enquanto as demais residências são omitidas. As informações geográficas que compõem o mapa possibilitam identificar outros aspectos secundários mas que, subjetivamente, influenciam na decisão de compra, como, por exemplo, o bairro onde a casa está localizada ou a existência de uma área verde nas proximidades. Ao colocar o ponteiro do mouse sobre um ponto colorido, o sistema apresenta informações detalhadas sobre a casa vinculada ao mesmo.

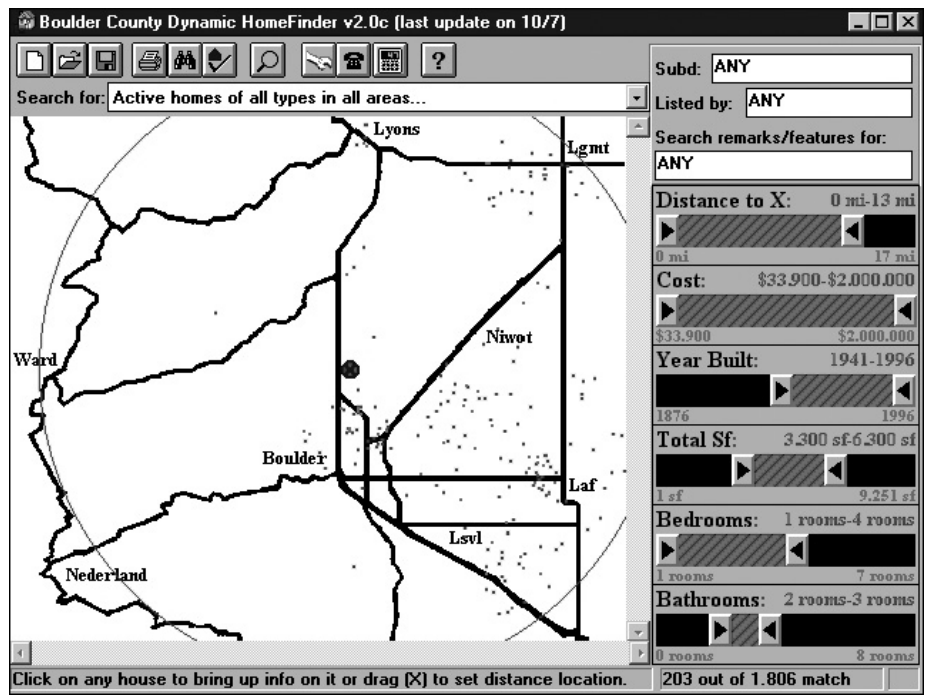

Outra categoria de técnicas de Visualização de Informações é composta por abordagens que variam a escala de ampliação da imagem. Uma técnica dessa categoria é a Foco+Contexto, a qual proporciona uma visão geral dos dados ao mesmo tempo em que destaca uma determinada região de interesse (foco) através de uma distorção da imagem. Diversas variações da técnica de Foco+Contexto existem, sendo algumas delas discutidas mais a frente.

Uma abordagem similar à técnica Foco+Contexto mas que não utiliza distorções é chamada de Overview+Detail.
Figura 4: Homefinder. Figura gerada utilizando o software demonstrativo em http://dq.com/ homefind/ 
Nessa abordagem, um subconjunto dos dados é apresentado de forma ampliada e detalhada em uma seção maior da visualização (o detail), enquanto que uma figura reduzida em uma posição lateral da tela (o overview) mostra uma visão geral de toda a informação disponível. A técnica mantém sincronizada a região ampliada com uma marca visual na figura reduzida, de forma a indicar qual parte dos dados está detalhada. Overview+Detail é muito empregada em processadores de texto e em programas de apresentação de slides e imagens para coordenar a navegação e a visualização de informações em uma lista de muitas páginas.

Além das formas já descritas de visualização, metáforas visuais vêm sendo empregadas como uma nova maneira de apresentar e explorar informações. Como exemplo, Panas e outros (PANAS; BERRIGAN; GRUNDY, 2003) propõem uma abordagem para visualizar software que mapeia linhas de código de programas de computador em prédios, quadras e ruas de uma cidade virtual tridimensional. Com esse tipo de visualização, é possível identificar o tamanho, a complexidade e outras características intrínsecas de um software e facilitar as atividades de melhoria e de evolução do mesmo através da utilização da capacidade intuitiva das pessoas para avaliar aspectos de tamanho, de mobilidade e de estética de uma cidade. Um trabalho similar é apresentado por Balzer e outros (BALZER et al., 2004), na qual estruturas estáticas de programas orientados a objetos são representadas como objetos tridimensionais colocados sobre um plano.

Nessa mesma linha das imagens metafóricas, outra abordagem interessante é a de paisagem (landscape), utilizada, por exemplo, para visualizar grandes volumes de documentos textuais com base em suas palavras-chave. A técnica consiste em desenhar um mapa de uma ilha de tal forma que cada palavra-chave (ou grupo de palavras-chave) é associada a uma única região do mesmo. A altura do relevo dessa região é diretamente proporcional à quantidade de documentos que contém as palavras-chave em questão. Além disso, se não há documentos que compartilham duas palavras-chave, então não existe uma linha reta de terra que liga as suas regiões vinculadas no mapa. A Figura 5 mostra uma tela do KartOO, um site de busca na Web que apresentava seus resultados na forma de landscapes interativos. O serviço do KartOO foi posteriormente interrompido.

Passando para o segundo critério apresentado no início dessa seção, as técnicas de visualização de informações po- 
dem seguir uma classificação de acordo com as características dos dados abstratos. Dados lineares e bidimensionais, por exemplo, podem ser visualizados através de várias técnicas como gráficos de pizza, de linha e de barra, Overview+Detail e Foco+Contexto.

Quando os dados possuem $n>3$ dimensões, técnicas mais elaboradas são necessárias. Uma dessas técnicas consiste em mapear apenas dois atributos dos dados para coordenadas $X, Y$ de um plano cartesiano e associar os demais atributos à forma, à cor e ao tamanho de objetos visuais. Outra solução é projetar o espaço $n$-dimensional no plano cartesiano, através de alguma técnica de projeção como a Multidimensional Scalling (BORG; GROENEN, 1997).

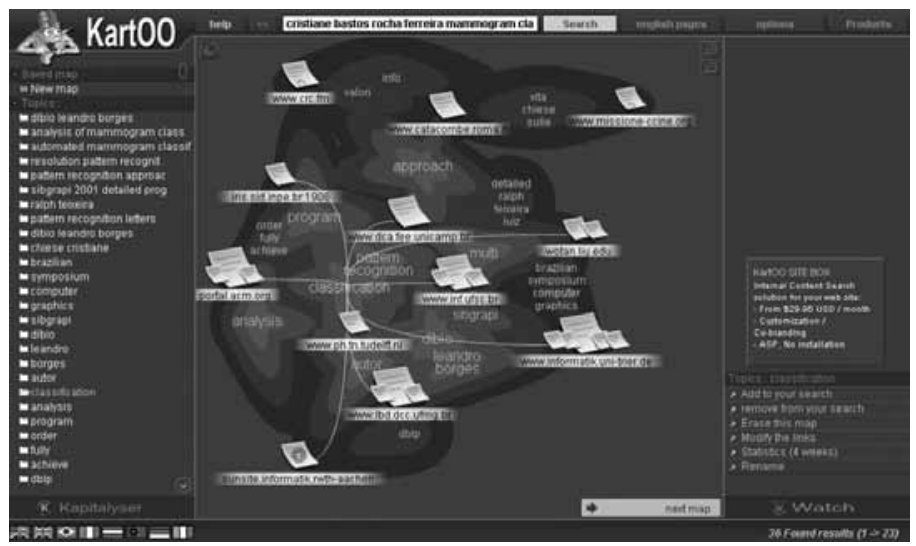

Duas outras técnicas de visualização muito utilizadas para dados multidimensionais são a de Coordenadas $\mathrm{Pa}$ ralelas e a de Glyphs. A técnica de Coordenadas Paralelas associa as dimensões dos dados abstratos a eixos paralelos verticais equidistantes, denominados coordenadas. Nessa técnica, cada dado é representado por uma linha atravessando os eixos em determinadas posições. Já os Glyphs (ou ícones) podem ser entendidos como uma representação simbólica que evidencia características essenciais de um dado ao qual se refere. Os Glyphs são construídos através de um mecanismo de codificação que associa atributos dos dados a propriedades visuais do ícone. Os detalhes de técnicas de Coordenadas Paralelas e de Glyphs são explicados nas próximas seções.

Se os dados abstratos representarem redes de relações entre objetos, uma forma efetiva de visualizá-los é construir
Figura 5: Exemplo de landscape usada no KartOO 
um grafo que descreva essa relação e gerar um desenho de sua estrutura. Grafos são modelos matemáticos formados por um conjunto de vértices e um conjunto de arestas. Os vértices podem representar objetos concretos ou abstratos quaisquer, enquanto as arestas indicam as relações entre pares desses objetos. Desenho de Grafos (DI BATTISTA et al., 1999) é uma das técnicas de visualização de informações mais comumente utilizadas para demonstrar relações entre objetos e pessoas e estruturas hierárquicas diversas. As técnicas de Desenho de Grafos têm aplicações, por exemplo, na microeletrônica para desenho de circuitos VLSI; na Engenharia de Software para representar componentes modulares de programas e a hierarquia de classes e de objetos (principalmente nas linguagens visuais e nas ferramentas de desenvolvimento de sistemas); em sistemas de telefonia para ilustrar uma rede de conexão telefônica ou até mesmo descrever chamadas telefônicas já realizadas em uma determinada região do país; e em ambientes de comunidade virtual para apresentar redes sociais. Atualmente, basta abrir um livro de ciências ou uma revista científica para perceber que as figuras ou diagramas ilustrativos têm um bom trabalho subjacente de desenho de grafos.

Relações hierárquicas entre dados podem ser visualizadas através de desenhos de grafos do tipo árvore, ao passo que outras formas de relações podem ser representadas por meio de desenho de grafos gerais ou de grafos direcionados.

A seguir são discutidos com mais detalhes algumas das técnicas de visualização de informações supracitadas.

\section{Foco+Contexto}

A ideia central das técnicas Foco+Contexto é apresentar uma visão geral dos dados a serem visualizados mas destacando uma região de interesse (foco) através de uma ampliação suave da mesma. Geralmente, procura-se ampliar a região de interesse enquanto se compacta o restante da imagem (o contexto). Como essa técnica emprega uma distorção da imagem original, ela pode exigir treinamento para seu uso eficaz.

Os principais exemplos de técnicas do tipo Foco+Contexto são a Fisheye, o Browser Hiperbólico, o Perspectiva Wall e o Table Lens. 


\section{Fisheye}

A técnica de visualização Fisheye, como o próprio nome diz, produz um efeito semelhante à lente de aumento de um olho de peixe, possibilitando uma visão detalhada de uma região de interesse através de uma taxa maior de ampliação no centro dessa região e decrescente no sentido da periferia da imagem.

Apesar do Fisheye ser intuitiva para imagens 2D, a técnica é muito mais geral e foi primeiramente proposta por Furnas (1981) como uma abordagem para visualização de texto. Na mesma, as seções de texto de menor interesse eram suprimidas, mostrando apenas o seu título. Já a seção ou subseção de interesse aparecia em detalhe.

A técnica foi utilizada ainda para navegação em árvores de diretórios, na qual subárvores eram expandidas ou compactadas de acordo com a localização de um ponto de interesse do leitor.

A Figura 6 mostra um exemplo de uma abordagem derivada do Fisheye aplicada a manipulação de menus, chamada de Fisheye menu (BEDERSON, 2000). A imagem à esquerda é uma abordagem tradicional de menu, onde uma barra de rolagem tem que ser utilizada para permitir acesso a todos os itens. Já a visualização à direita emprega o conceito de Fisheye. Nesta segunda imagem, todos os itens são apresentados simultaneamente, sendo permitida a movimentação do ponteiro do mouse para destacar um ponto de foco (indicado em cor mais escura), o que causa uma ampliação gradual dos itens próximos a ele.

\begin{tabular}{l}
\hline About Portal \\
AltaVista Search \\
Amazon Shopping \\
Andale Shopping \\
Any Day Calendar \\
AOL Instant Messager \\
Ask Jeeves \\
Auctions \\
BigStep Small Business \\
BizRate Surveys \\
BizTravel \\
Brittanica \\
C|Net Technology \\
Carnegie Mellon University \\
CBS Sportsline \\
CenterBeam Business \\
Charles Schwab \\
Chumbo Computer Shopping \\
DealTime Shopping \\
Deep Canyon Research \\
Deja Opinions \\
Dell Computer \\
DevEdge Web Development \\
DirectHit Search \\
Discovery Channel for Kids \\
Disney \\
-n
\end{tabular}

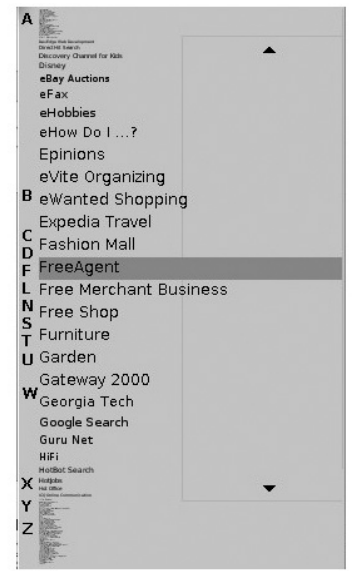

Figure 6: Fisheye menu (BEDERSON, 200o). Applet Java disponível em http://www.cs.umd.edu/ hcil/fisheyemenu/ 


\section{Browser Hiperbólico}

O Browser Hiperbólico combina Foco+Contexto com desenho radial de árvores para auxiliar na exploração de grandes hierarquias. Um dos principais trabalhos com essa técnica é a proposta de Lamping e Rao (1996), na qual a estrutura de uma árvore é mapeada em uma representação no plano hiperbólico a ser projetado na tela. Uma propriedade conveniente desse plano é que a circunferência de um círculo cujo centro coincide com o centro da tela cresce exponencialmente com o seu raio. Isso significa que há mais espaço disponível para se compactar informações na medida em que se aproxima da periferia do desenho.

Com essa representação, o Browser Hiperbólico consegue disponibilizar cerca de dez vezes mais vértices de uma árvore do que utilizando uma visualização no plano cartesiano.

A navegação em um Browser Hiperbólico é também mais efetiva. Mudanças de foco podem ser realizadas através de movimentos simples do mouse objetivando selecionar vértices de maior interesse. Isso move o vértice selecionado para o centro da tela e ocasiona a compactação das informações que estão distantes do mesmo. Outrossim, o sistema realiza transições gradativas e suaves de mudança de ponto de foco, a fim de preservar o mapa mental.

Uma ilustração de Browser Hiperbólico é apresentada na Figura 7.

Figura 7: Exemplo de Browser Hiperbólico da Inxight Software, Inc. Applet disponível em http:// www.flashkit.com/search/sitemap/index.shtml.

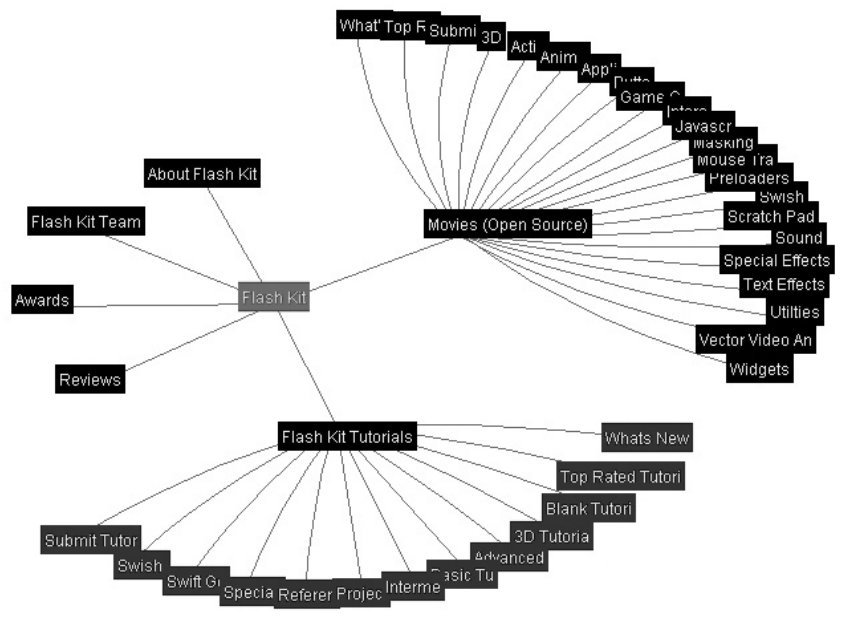




\section{Perspective Wall e Table Lens}

A técnica Perspective Wall (MACKINLAY; ROBERTSON; CARD, 1991) permite a visualização de muitas informações sequencias em um retângulo horizontal, o qual é dobrado para trás nos limites à direita e à esquerda da região de interesse, fornecendo, assim, uma perspectiva $3 \mathrm{D}$ do resultado. Uma ilustração dessa técnica pode ser vista na Figura 8.

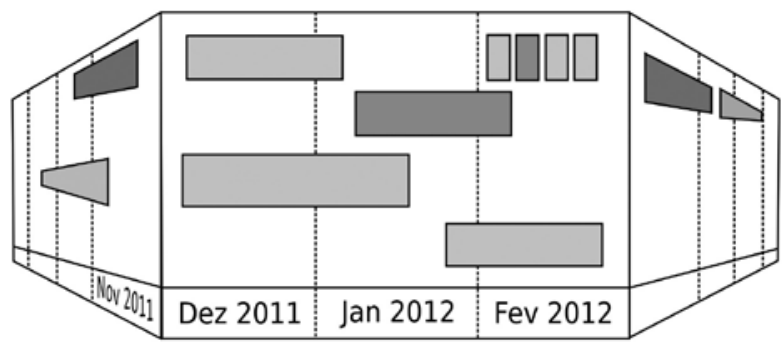

Já a técnica Table Lens (RAO; CARD, 1994) é uma abordagem efetiva para o entendimento de grandes volumes de dados numéricos e categóricos multidimensionais dispostos em uma tabela e apresentados integralmente em uma única tela. Os dados (linhas) de maior interesse aparecem expandidos, enquanto os demais itens são compactados como finas linhas de pixel. O comprimento das linhas de pixel é determinado pelo atributo do dado na coluna correspondente. A técnica possibilita interação do usuário visando selecionar as linhas de interesse, o que causa a ampliação das mesmas e a apresentação dos valores exatos nas suas colunas. É possível também ordenar as linhas com base nos atributos das colunas para facilitar a consulta dos dados e identificar correlações. A Figura 9 demonstra uma Table Lens.

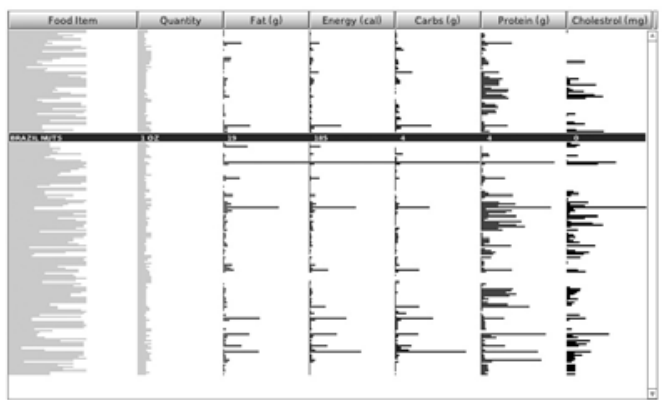

Figura 8: Calendário apresentado usando a técnica Perspective Wall. É possivel avançar ou recuar no calendário, sendo que os três meses consecutivos em foco aparecem ampliados no centro da tela.

Figure 9: Visualização de Informações usando a técnica Table Lens. 


\subsection{Coordenadas Paralelas}

A técnica de Coordenadas Paralelas foi proposta por Inselberg (INSELBERG; DIMSDALE, 1990; INSELBERG, 1997). Ela mapeia um espaço $n$-dimensional em uma estrutura bidimensional que usa $n$ eixos paralelos verticais equidistantes, denominados coordenadas.

Os eixos verticais representam as dimensões ou atributos dos dados. Uma linha gráfica associada a cada item de dado conecta os eixos nos seus respectivos valores, o que permite a observação de padrões.

A Figura 10, gerada a partir da ferramenta VisEd (XAVIER, 2004), demonstra um exemplo dessa técnica. A VisEd é uma ferramenta desenvolvida na Universidade Federal de Goiás para apoio ao ensino à distância via $\mathrm{Web}$, permitindo a visualização de dados sobre a participação e o desempenho de alunos em um determinado curso. Na figura, os eixos verticais representam os seguintes atributos dos alunos: nome, idade, bairro onde mora, número de acessos ao chat do curso e nota final. Foi selecionada, pelo usuário da ferramenta, a faixa de variação de valores entre 80 e 100 no eixo do atributo Nota, o que ocasionou o destaque das linhas correspondentes aos alunos que tiraram notas neste intervalo, facilitando, assim, a visualização dos respectivos valores nos outros eixos.

As formas de interação oferecidas pela técnica de Coordenadas Paralelas consistem em:

- inclusão ou remoção de eixos (coordenadas);

- seleção de um intervalo de interesse em um eixo. Nesse caso, as linhas que passam pelo intervalo selecionado são destacadas com uma cor mais intensa, enquanto as demais têm a sua cor atenuada, conforme pode ser visto na Figura 10. Esse processo simboliza uma filtragem simples dos dados;

- seleção de intervalos em duas ou mais coordenadas simultaneamente. Tal processo permite a criação de filtros complexos para os dados, definindo operações como união, intersecção ou complemento entre os conjuntos filtrados de cada coordenada. Por exemplo, na Figura 10, poderia ser definida uma consulta para evidenciar os alunos com idade entre 22 e 28 anos e que residem nos bairros "Sudoeste" e "Aeroporto" de Goiânia, GO. 


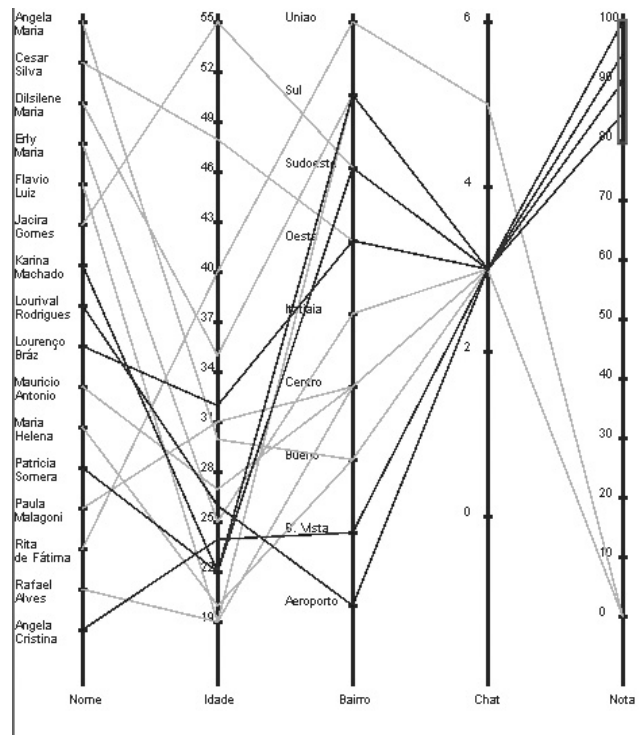

Coordenadas Paralelas são adequadas para mineração de dados com várias dimensões pois permitem transformar a busca por relações entre pares de atributos em um processo mais intuitivo, de reconhecimento de padrões $2 \mathrm{D}$. A técnica enfatiza, principalmente, relações entre eixos adjacentes e conjuntos de dados que possuem padrões visuais similares.

Pode-se realizar ainda a normalização do intervalo de valores de cada coordenada de maneira individual ou em grupo, objetivando realçar os padrões visuais. Outro parâmetro que pode ser configurado para aumentar efetividade é a ordem com que as coordenadas são apresentadas, o que implica em mais ou menos cruzamentos entre linhas. Normalmente, essa ordem é sugerida pelos próprios atributos dos dados.

Vários trabalhos têm sido desenvolvidos no sentido de estender a técnica básica de Coordenadas Paralelas (ARTERO; DE OLIVEIRA; LEVKOWITZ, 2004), demonstrando que, apesar de simples, as formas de utilização dessa abordagem ainda não se esgotaram.

\subsection{Glyphs}

Outra classe de técnicas de Visualização de Informações para dados multidimensionais está relacionada com o uso de Glyphs, também chamados de ícones. Os Glyphs possuem atributos geométricos, tais como forma, tamanho, orientação,
Figura 10: Exemplo de visualização usando a técnica Coordenadas Paralelas, gerada pela Ferramenta VisEd (XAVIER, 2004). 
posição ou direção, e atributos de aparência, como cor, textura e transparência. Cada glyph ou ícone pode ser associado a um dado diferente, possibilitando, assim, uma visualização rápida e compacta de vários ícones simultaneamente representando todo o conjunto de dados.

Um dos primeiros trabalhos nesse tema foi apresentado por Chernoff (1973), onde os atributos visuais dos ícones foram explorados de um modo bastante particular. Chernoff observou que os seres humanos são muito sensíveis a uma variedade de expressões faciais. Ele então sugeriu a utilização de ícones representando faces, na qual cada característica como o tamanho dos olhos, a altura da sobrancelha e a forma da boca pudesse ser associada a um atributo de dado. Chernoff aplicou sua técnica para estudar exemplos geológicos, com cada face cobrindo dezoito atributos. Sua técnica ficou conhecida como Faces de Chernoff.

A Figura 11 apresenta uma visualização baseada nas Faces de Chernoff, gerada novamente pela ferramenta VisEd. Os dados dos alunos como sexo, quantidade de acesso ao sito do curso e nota final foram mapeados em atributos visuais de um rosto. O mapeamento visual, realizado para o exemplo em questão, consistiu em vincular o número de acessos à quantidade de cabelo sobre o rosto, a nota final do aluno à inclinação da boca (triste, séria ou feliz) e o sexo como a cor da face. Essas associações são totalmente flexíveis, podendo ser modificadas dinamicamente através de uma seleção simples para cada atributo. Além disso, é possível ordenar as faces com base em um atributo específico, buscando enfatizar um determinado padrão. Os ícones da Figura 11, por exemplo, foram classificados por ordem decrescente de nota final. Através da análise dessa imagem, verifica-se que os alunos que obtiveram notas mais baixas (faces da última linha da figura) também acessaram pouco o site do curso (quantidade menor de cabelo).

Figura 11: Visualização de dados de alunos utilizando Faces de Chernoff. Imagem gerada pela Ferramenta VisEd (XAVIER, 2004).
Ferramenta DisED

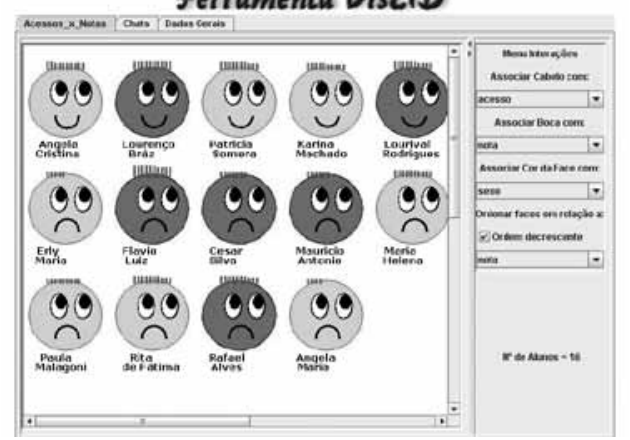


A quantidade de ícones que podem ser apresentados ao mesmo tempo em uma visualização baseada em Glyphs de maneira efetiva depende, principalmente do tamanho da tela e do formato dos ícones. Em geral, existe um conflito entre mostrar muitos ícones e apresentá-los com muitos detalhes (atributos visuais). Se vários ícones têm que ser mostrados simultaneamente, então o tamanho dos mesmos precisa ser reduzido, o que impede a percepção de algumas características sutis como, por exemplo, a inclinação da sobrancelha de uma face. Por outro lado, se os ícones tiverem muitos detalhes, então mais espaço na tela será necessário para desenhar os mesmos, o que reduz a quantidade ícones que podem ser mostrados ao mesmo tempo. A escolha do tamanho ideal dos ícones é uma decisão que pode ser deixada para o próprio usuário, através da implementação de ferramentas interativas de zoom.

As principais formas de interação com uma visualização baseada em Glyphs são, portanto:

- modificação dinâmica da associação dos atributos dos dados com os atributos visuais dos ícones;

- alteração do tamanho dos ícones, objetivando controlar a quantidade total de ícones a serem visualizados simultaneamente na tela;

- ordenação dos ícones de acordo com os atributos desejados; e

- acesso a informações mais detalhadas sobre os dados relacionados com um ícone através de um clique sobre o mesmo.

A visualização usando Glyphs é bastante motivadora. Entretanto, esse tipo de técnica exige um tempo maior para treinamento do usuário, visto que é preciso habituar-se a interpretar cada atributo visual do ícone de acordo com o mapeamento visual adotado.

\section{StarPlot}

A técnica de visualização Starplot (LEE; REILLY; BUTAVICIUS, 2003) combina Glyphs com Coordenadas Paralelas, sendo voltada também para a visualização de dados multidimensionais. Na Starplot, os eixos, que na técnica de Coordenadas Paralelas eram paralelos, agora partem de uma origem comum e irradiam na forma de estrela. Cada linha de dado da tabela é representado por uma estrela e várias estreladas podem ser apresentadas simultaneamente na tela para comparação entre si. 
Um exemplo dessa técnica é ilustrado na Figura 12 para visualização da produção acadêmica de docentes de uma universidade. No exemplo, cada ícone-estrela representa um docente. As pontas das estrelas são os tipos de atividades acadêmicas e o seu comprimento é dado pela quantidade de produção naquele tipo de atividade.

\subsection{Tree-Maps}

Johnson e Shneiderman (1991) propõem uma técnica denominada Tree-Map para visualizar árvores hierárquicas cujos elementos possuem atributos valorados acumulados na medida que se caminha das folhas para a raiz. A técnica consiste em representar o nível mais alto da hierarquia (a raiz) como uma região retangular que preenche todo o espaço de desenho. Os níveis mais baixos são desenhados recursivamente como retângulos dentro da região maior. O tamanho de cada retângulo é proporcional aos valores de itens nos níveis imediatamente abaixo na hierarquia.

A Figura 13 ilustra a aplicação dessa técnica para visualizar o espaço consumido por arquivos e pastas e em um sistema de arquivos no computador. Quanto maior é o tamanho dos arquivos dentro de uma pasta, maior é a região retangular vinculada à mesma.

Figure 12: Visualização de dados de produção acadêmica de docentes usando Starplots.

Figura 13: Exemplo da Técnica Tree-map para visualizar uma árvore de diretórios. O retângulo maior, envolvendo toda a figura, representa a raiz da árvore e, portanto, o espaço consumido por todos os seus arquivos $e$ subpastas. Os demais retângulos, internos, representam as subpastas.
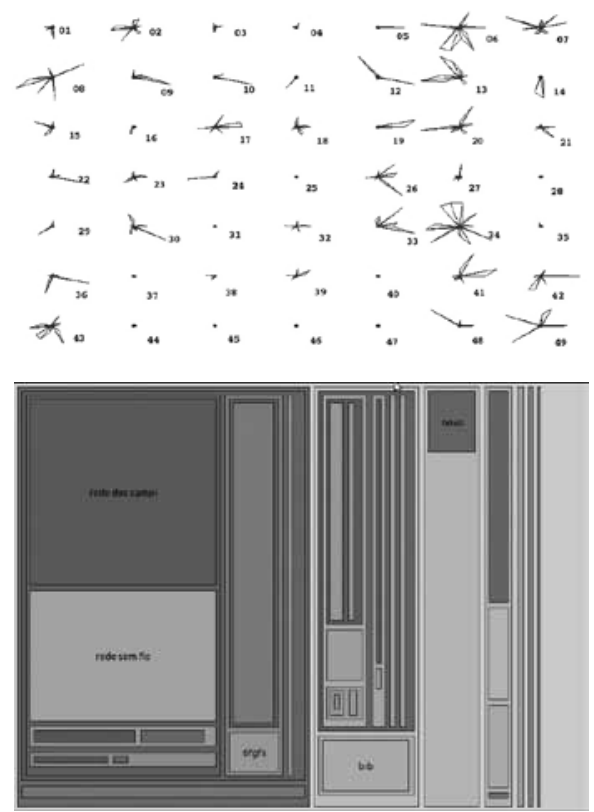


\section{Conclusão}

Neste trabalho, apresentamos uma revisão geral da área de Visualização de Informações e explicamos a sua importância para a compreensão das informações subjacentes a dados abstratos. Discutimos também algumas técnicas de visualização de informações bastante utilizadas e apresentamos sugestões para tornar as visualizações mais expressivas e efetivas.

Existem várias outras técnicas de representação visual de informações que não foram abordadas no presente artigo. Há também um esforço científico intenso no sentido de criar novas técnicas e ferramentas computacionais de apoio a essa área ou de demonstrar novas aplicações práticas das técnicas já existentes.

Entre os desafios mais modernos na Visualização de Informações, destacam-se aqueles que surgem da necessidade de manter a constante atualização dos conhecimentos e das técnicas da área com os novos e populares dispositivos de visualização e de interação humano-computador. Assim, podemos citar as seguintes necessidades:

- a adequação das técnicas de visualização de informações para celulares e tablets. Com a popularização desses dispositivos, há um interesse cada vez maior em disponibilizar nos mesmos as aplicações que originalmente foram desenvolvidas para computadores maiores. Apesar do ganho em mobilidade, tais equipamentos infelizmente apresentam dois fortes obstáculos à visualização de informações. O primeiro é a baixa resolução de sua tela (em quantidade de pixels), em geral muito inferior do que a de um monitor comum, o que pode implicar em uma menor quantidade de dados a ser mostrados e, portanto, em uma baixa expressividade. O outro fator diz respeito ao próprio tamanho reduzido da tela, o qual dificulta a percepção e a interação com detalhes da imagem, mesmo se apresentada em um espaço de alta definição. Esse dois obstáculos exigem dividir a visualização de em pedaços menores e implementar mecanismos para recuperar e apresentar os mesmos. Com isso, o esforço cognitivo para compreensão da base de dados é aumentado e a efetividade fica comprometida. Uma sugestão visando compensar tais limitações seria utilizar técnicas de visualização que integram, em uma mesma imagem, uma visão global e uma região ampliada dos dados, como é o caso da Overview+Detail e da 
Foco+Contexto. Animações também poderiam ser empregadas para garantir uma transição suave da imagem e, desta forma, preservar o mapa mental quando o usuário navegasse na visualização.

- o desenvolvimento de técnicas de visualização de informações próprias para ambientes de telas grandes e de alta definição, acoplando dois ou mais projetores ou displays. Tais ambientes são mais caros, possuem pouca ou nenhuma mobilidade e têm baixa disponibilidade. Mesmo assim, eles estão se tornando comuns em atividades colaborativas nos centros de pesquisas, nas empresas e em organizações governamentais, o que justifica o desenvolvimento de novas formas de visualização que explorem as características peculiares dos mesmos. Entre as vantagens desses ambientes destacam-se: permitirem visualizações mais expressivas em função da disponibilidade de mais pixels; e reduzirem a distração com eventos alheios à tarefa de visualização por ocupar uma grande porção do campo de visão do observador (DO NASCIMENTO, 2003).

- a combinação da visualização com outros canais de transmissão de dados perceptíveis aos seres humanos, como a audição e o tato. O processo, por exemplo, de "sonorificação" de dados consiste em associar a amplitude e a frequência de ondas sonoras a um conjunto de atributos dos dados. Em seguida, define-se um caminho de varredura através do qual os dados são consultados e os seus sons correspondentes produzidos. Já a sensação de tato é comumente implementada por meio de luvas sensoriais ou através de um dispositivo denominado Haptic (MASSIE; SALISBURG, 1994). O Haptic consiste em um objeto real que pode ser movido pelo usuário com certo grau de liberdade. O movimento é reconhecido pelo computador e forças podem ser aplicadas ao objeto para dar a sensação de interação com itens virtuais associados ao mesmo, em alguns casos, dificultando o próprio movimento. É possível, assim, explorar uma base de dados mapeando-a visualmente para um ambiente virtual e permitindo ao usuário navegar por esse espaço fisicamente sentindo as suas "peculiaridades". Uma investigação inicial sobre o uso de displays com multissensores foi feita por Nesbitt (2003).

- Um estudo mais detalhado de técnicas de visualização tridimensional de informações e de sua efetividade e 
expressividade quando comparadas à visualizações $2 D$. Certamente, a percepção de uma imagem tridimensional é intuitiva e ajuda na compreensão de dados quando os mesmos já possuem uma representação geométrica intrínseca em três dimensões; isso vale também para as imagens $3 \mathrm{D}$ projetadas em um plano. No entanto, ainda não se tem um compreensão de todas as regras e condições que definem quando uma visualização $3 \mathrm{D}$ é melhor do que uma $2 \mathrm{D}$.

\section{Referências}

AHLBERG, C; SHNEIDERMAN, B. Visual information seeking: Tight coupling of dynamic query filters with starfield displays. Proc. of Human Factors in Computing Systems, CHI'94, 1994, p. 313-317.

ARTERO, A. O.; DE OLIVEIRA, M. C. F.; LEVKOWITZ, H. Uncovering clusters in crowded parallel coordinates visualizations. Proceedings of the IEEE Symposium on Information Visualization (INFOVIS'o4), Washington, DC, USA: IEEE Computer Society, 2004, p. 81-88.

BALZER, M.; NOACK, A.; DEUSSEN, O.; LEWERENTZ, C. Software landscapes: Visualizing the structure of large software systems. Proc. of Symposium on Visualization (VisSym), Konstanz, Germany, 2004, p. 261-266, .

BEDERSON, B. B. Fisheye Menus. Proceedings of ACM Conference on User Interface Software and Technology (UIST 2000), ACM Press, 2000, p. 217-226.

BORG, I.; GROENEN, P. Modern Multidimensional Scaling. New York: Springer-Verlag, 1997.

CARD, S. K.; MACKINLAY, J. D.; SHNEIDERMAN, B.; CARD, M. Readings in Information Visualization: Using Vision to Think. Morgan Kaufmann Series in Interactive Technologies, Academic Press, 1999.

CHERNOFF, $\mathrm{H}$. The use of faces to represent points in k-dimensional space graphically. Journal of the American Statistical Association, 1973, p. 361-368.

CLEVELAND, W. S.; MCGILL, R. Graphical perception: Theory, experimentation and application to the development of graphical methods. J. American Statistical Association. 79(387), 1984, p. 531-554.

DI BATTISTA, G.; EADES, P.; TAMASSIA, R.; TOLLIS, I. G. Graph Drawing. Upper Saddle River, NJ: Prentice Hall, 1999. 
DO NASCIMENTO, H. A. D. User Hints for Optimization Processes. 2003. PhD Thesis - University of Sydney, Australia, 2003.

DWYER, T.; EADES, P. Visualising a fund manager flow graph with columns and worms. In Proceedings of the 6th International Conference on Information Visualisation (IVo2), IEEE Computer Society, 2002, p. 147-158.

FURNAS, G. W. The FISHEYE view: A new look at structured files. Technical Report \#81-11221-9, Bell Laboratories, Murray Hill, New Jersey 07974, U.S.A, 1981.

INSELBERG, A. Multidimensional detective. In IEEE Symposium on Information Visualization (InfoVis '97), Washington - Brussels - Tokyo: IEEE, 1997, p. 100-107.

INSELBERG, A.; DIMSDALE, B. Parallel coordinates: a tool for visualizing multi-dimensional geometry. In Proceedings of the ist Conference on Visualization (VIS '9o), Los Alamitos, CA, USA: IEEE Computer Society Press, 1990, p. 361-378.

JOHNSON, B.; SHNEIDERMAN, B. Tree-maps: a space-filling approach to the visualization of hierarchical information structures. In Proceedings of the and Conference on Visualization (VIS '91), IEEE Computer Society Press, 1991, p. 284-291.

LAMPING, J.; RAO, R. The hyperbolic browser: A focus + context technique for visualizing large hierarchies. Journal of Visual Languages and Computing, 7(1), 1996, p. 33-35.

LEE, M. D.; REILLY, R. E.; BUTAVICIUS, M. E. An empirical evaluation of chernoff faces, star glyphs, and spatial visualizations for binary data. In Proceedings of the Australian symposium on Information visualisation (CRPITS '24), Darlinghurst, Australia: Australian Computer Society, Inc, 2003, p. 1-10.

MACKINLAY, J. Automating the design of graphical presentations of relational information. ACM Transactions on Graphics, 5(2), 1986, p. 110-141.

MACKINLAY, J. D.; ROBERTSON, G. G.; CARD, C. The perspective wall: Detail and context smoothly integrated. In Proceedings of Conference on Human Factors in Computing Systems and Graphics Interface, ACM-Press, 1991, p. 173-179.

MARR, D. Vision - A Computational Investigation into the Human Representation and Processing of Visual Information. W. H. Freeman and Company, 1982.

MASSIE, T. H.; SALISBURG, J. K. The phantom haptic interface: A device for probing virtual objects. In Proceedings 
of the ASME International Mechanical Engineering Congress and Exhibition, Chicago, IL, volume DSC 55-1, 1994, p. 295-302.

NESBITT, K. V. Multi-sensory Display of Abstract Data. 2003. PhD Thesis, School of Information Technologies, University of Sydney, Australia, 2003.

PANAS, T.; BERRIGAN, R.; GRUNDY, J. A 3D Metaphor for Software Production Visualization. In Proc. of Seventh International Conference on Information Visualization (IV'o3), 2003, p. 314-319.

RAO, R.; CARD, S. K. The table lens: Merging graphical and symbolic representation in an interactive focus + context visualization for tabular information. In Proc. ACM Conf. on Human Factors in Software, ACM-Press, 1994, p. 318322.

SPENCE, R. Information Visualization. ACM Press, 2000.

TUFTE, E. R. The Visual Display of Quantitative Information. CT: Graphics Press, 1983.

TUFTE, E. R. Envisioning Information. Graphics Press, 1990.

TUFTE, E. R. Visual explanations: images and quantities, evidence and narrative. Graphics Press, 1997.

WARE, C. Information Visualization: Perception for Design. $4^{\text {th edition, Morgan Kaufmann Interactive Technologies }}$ Series, 2004.

WILLIAMSON, C.; SHNEIDERMAN, B. The dynamic homefinder: evaluating dynamic queries in a real-estate information exploration system. In Proceedings of the 15th Annual International ACM SIGIR conference on Research and development in information retrieval (SIGIR '92), New York, NY, USA: ACM Press, 1992, p. 338-346.

XAVIER, S. S. A. Visualizações de informação para suporte a EAD - ferramenta VisED. Monografia de Projeto Final de Curso, Instituto de Informática, Universidade Federal de Goiás, 2004.

Recebido em: 05/01/12

Aceito em: 05/01/12 


\section{HUGO A. D. DO NASCIMENTO}

hadn@inf.ufg.br

Bolsista de Produtividade em Pesquisa 2 do CNPq, possui graduação em Ciências da Computação pela Universidade Federal do Rio Grande do Norte (1994), mestrado em Ciência da Computação pela Universidade Estadual de Campinas (1997) e doutorado em Computer Science pela University of Sydney (2003). Atualmente é Professor Adjunto Nível 3 da Universidade Federal de Goiás, no Instituto de Informática.

\section{CRISTIANE B. R. FERREIRA cristiane@inf.ufg.br Bacharela em Ciências da Computação pela Universidade Federal de Goiás (UFG) e Mestre em Informática Aplicada pela Pontifí- cia Universidade Católica do Paraná (PUC-PR). Desde de 2002, Cristiane é professora do Instituto de Informática da UFG e vem trabalhando nas áreas de Análise Multirresolução, Percepção, Re- conhecimento de Padrões, Visão Computacional e Processamento de Imagens.}

